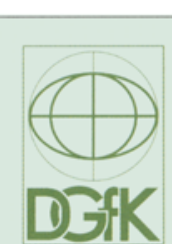

KN Kartographische Nachrichten Journal of Cartography and Geographic Information 67. Jahrgang, Juni 2017, Heft 3

Herausgeber: Deutsche Gesellschaft für Kartographie e.V. (DGfK) - Gesellschaft für Kartographie und Geomatik, vertreten durch den Präsidenten Prof. Dr. Manfred Weisensee

Organ der Deutschen Gesellschaft für Kartographie e. V. (DGFK), der Schweizerischen Gesellschaft für Kartografie (SGK) und der Österreichischen Kartographischen Kommission (OKK) in der

Österreichischen Gesellschaft für Geographie (ÖGG).

Schriftleitung

Hauptschriftleiter

Prof. Dr. rer. nat. habil. Mark Vetter (zuständig für Aufsätze. Berichte). Hochschule Karlsruhe - Technik und Wirtschaft,

Molikestr. 30, 76133 Karlsruhe; redaktion@kartographische-

nachrichten.de, Fon:0049(0) 7219252599

Weitere Schriftleiter

Prof. Dr. phil. Frank Dickmann (Zweiter Schriftleiter, zuständig für Nachrichten aus Hochschulen und Institutionen, Rezensionen.

Ruhr-Universităt Bochum, Geographisches Institut,

Postfach, 44780 Bochum; frank.dickmann @rub.de

Fon: 0049(0) 234 32-23379; Fax: 0049(0) 234 32-14964

Dipl.-Ing. Andreas Gollenstede (zuständig für Geoinformation

aktuell) Haareneschstr. 91, 26121 Oldenburg; kn@gollenstede.com

Fon: 0049(0) 441 7779-545; Fax: 0049(0) 441 7779-750

Dr.-Ing. Thomas Chudy (zuständig für DGfK-, SKG- und ÖGG-

Nachrichten). Martin-Luther-Universität Halle-Wittenberg, Institu

fur Agrar- und Ernăhrungswissenschaften, Karl-Freinerr-von-
Fritsch-Straße 4,06120 Halle/Saale, Fon:0049(0) 3455522448 ;

Fritsch-Straße 4, 06120 Ha $0049(0) 3455527109$

Prof. Dr. Reinhard Zölitz (zuständig für Nachrichten aus Hochschulen und Institutionen), Institut für Geographie und Geologie, Kartographie und GIS, Friedrich-Ludwig-Jahn-Str. 16 D-17489 Greifswald, Fon 03834864523 , Fax: 03834864501 .

E-Mail:zoelitz@uni-greifswald de

Mitarbeit für die Schweiz: Prof. Dr. Lorenz Hurni. Institut für Kartografie, ETH Hönggerberg, 8093 Zürich; hurni@karto.baug.eth2 ch. Fon: 0041 16333033; Fax: 00411631153

Mitarbeit für Österreich: Univ.-Prof. Dr. Wolfgang Kainz Institut für Geographie und Regionalforschung, Universitāt Wien Universitätsstraße 7/5, 1010 Wien; wolfgang.kainz@univie.ac.at Fon: 0043 14277-8640; Fax: 0043 14277-9531

Ehrenschriftleiter

Prof. i. R. Dr. Jürgen Dodt t, Witten

Dr, rer nat. h.c. Rolf Harbeck, Bonn

Editorial Board

Prof. Dr. H. Asche, Potsdam; Prof. Dr. Manfred Buchroithner

Dresden; Prof. Dr. Dirk Burghardt, Dresden; Prof. i. R. Dr. Jürgen

Dodt, Witten; Prof. Dr. D. Dransch, Potsdam; Prof. Dr. M. Ehlers,

Osnabrück, Berlin; Prof. Dr. S. I. Fabrikant, Zürich, Schweiz;

Ass.-Prof. Dr. S. Fuhrmann, Fairfax, USA; Prof. Dr. G. Gartner, Wien, Osterreich; Prof. Dr. D. Grünreich, Ronnenberg; Dr. Rolf Harbeck Bonn: Prof. Dr. A. Hüttermann. Ludwigsburg: Prof. Dr. L Hurni, Zürich: Prof. Dr.W. Kainz, Wien: Prof. Dr. P. Kammere München; Zurich, Prof. Dr.W. Kainz, Wien; Prof. Dr.P. Kammerer, Munchen: Prof. Dr. W. G. Koch, Dresden; Prof. Dr. Menno-Jan Kraak, Twente,
Netherlands; Prof. Dr. Jukka Krisp, Augsburg; Prof. Dr.-Ing.

Netherlands; Prof. Dr. Jukka Krisp, Augsburg; Prof. Dr.-Ing.
L Meng, München; Prof. Dr. J. Schiewe, Hamburg; PD Dr.-Ing

J. Schoppmeyer, Bonn; Prof. Dr.J. Schweikart, Berlin; Prof.

Dr.-Ing. M. Sester, Hannover; Prof. Dr. J. Siemer, Regina, Kanada;

Prof. Dr. R. Zolitz, Greifswald.

Manuskripte richten Sie bitte an den zuständigen Schriftleiter.

Hinweise zur Gestaltung des Manuskripts finden Sie unter

www.kartographische-nachrichten.de > Autorenhinweise.

Reviewverfahren

Wissenschaftliche Beiträge werden im Rahmen eines Peer-ReviewVerfahrens begutachtet. Trotz sorgfáltiger Redigierung übernehmen Herausgeber, Redaktion und Verlag keine Haftung für die inhaltliche Richtigkeit der Veröffentlichungen.

Die KN Kartographische Nachrichten ist in der internationalen

Zitationsdatenbank Scopus gelistet.

Die DGFK ist Mitglied der

Internationalen Kartographischen

Vereinigung IKV/ICA.

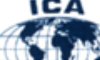

Verlag: Kirschbaum Verlag GmbH, Siegfriedstraße 28,

D-53179 Bonn / Postfach 210209 , D-53157 Bonn,

Fon: (0228) 95453-0. Fax: (0228) 95453-27:

Internet: http://www.kirschbaum.de, E-Mail: info@kirschbaum.de

Anzeigenleiter: Volker Rutkowski; zurzeit ist Anzeigenpreisliste

Nr. 52 vom 1.10.2016 gültig

Bezugspreise und ISSN: Inland/Ausland Jahresabonnement

inkl. E-Paper und elektronischem Archiv 74,- Euro zzg.

Versand 9,80,- Euro (jeweils einschl. MwSt).

Einzelheft 18,- Euro zzgl. Versand

Die Zeitschrift erscheint alle zwei Monate. ISSN 0022-9164

Kündigungsfrist 6 Wochen zum Auslaufen des Abonnement-

Zeitraumes.

Satz: EMS Eckert Medienservice, 53359 Rheinbach

Druck: johnen-druck GmbH \& CO. KG, Industriegebiet Bornwiese.

54470 Bernkastel-Kues

\title{
Lingua Franca English
}

\section{Liebe Kolleginnen und Kollegen,}

mit Bestürzung hat die Redaktion vom Tode Jürgen Dodts erfahren. Wir trauern um unseren Kollegen und Ehrenschriftleiter, der fast zwanzig Jahre lang die Kartographischen Nachrichten als Hauptschriftleiter betreut und geprägt hat. Rolf Harbeck würdigt in diesem Heft (S. 142) das Wirken Jürgen Dodts in einem Nachruf.

Unsere Kartographischen Nachrichten haben als Fachzeitschrift eine lange Tradition. Die Zeitschrift ist in Deutschland entstanden und hatte in den letzten Jahrzehnten in Mitteleuropa eine herausragende Bedeutung als Gradmesser für den aktuellen Stand der Kartographie und auch als Impulsgeber für die zukünftige Kartographie. Es

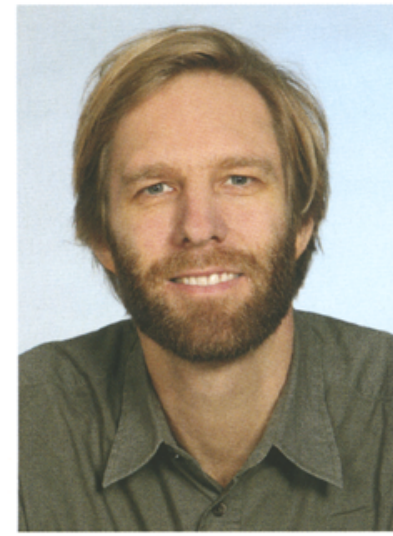

Mark Vetter ist eine große Ehre für den Hauptschriftleiter, wenn man diese Tradition fortsetzen darf.

Es ist aber auch eine Verpflichtung. Eine Verpflichtung, die man gerne annimmt und auch verantwortungsbewusst umsetzen sollte. Was bedeutet diese Verpflichtung? Aus meiner Sicht ist das Hauptziel selbstverständlich die Stärkung der Reputation der Zeitschrift und die Sicherstellung der Zukunftsfähigkeit des Journals. Bundesligatrainer würden mindestens vom Klassenerhalt sprechen.

Wie gelingt dieser Klassenerhalt? Für mich gibt es hier nur eine Lösung, um zu verhindern, dass unser Journal nicht eines Tages in der Bedeutungslosigkeit verschwindet: Wir müssen für eine hohe Attraktivität zur Veröffentlichung von Beiträgen in unserer Zeitschrift sorgen. Und wir müssen für eine hohe Verbreitung der wissenschaftlichen Erkenntnisse sorgen. Was ist das höchste Ziel einer Wissenschaftlerin, eines Wissenschaftlers? Maximale Verbreitung ihrer/seiner Ideen. Dies können wir nur mit englischen Beiträgen erreichen, die somit auch weltweit - am besten online - von jederfrau/jedermann aus der scientific community gelesen werden können.

Somit werden wir in Zukunft immer mehr Fachartikel in englischer Sprache in den KN lesen müssen. Dieser Weg ist alternativlos. Aus meiner Sicht. Gerne freue ich mich auf Ihre Meinungsäußerung und auch über Ihr Engagement für etwaige Alternativen!

Dennoch, und dies ist mir sehr wichtig zu betonen: Das Vereinsleben findet selbstverständlich weiterhin auf Deutsch statt. Es ist überhaupt nicht unser Ziel, den Sektions-, den Fachberichts-, den Nachrichten-, den Leserbrief- oder den Ereignisteil auf Englisch zu publizieren. Insbesondere bitte ich Sie, über die Möglichkeit der Veröffentlichung eines Fachberichtes auf Deutsch über die Kartographie in Anwendung/Praxis nachzudenken! Diese Möglichkeit steht jeder/jedem offen und dies wird so bleiben!

Im vorliegenden Heft geht es zweimal explizit um das Thema Kartenprojektion, beide Artikel sind auf Deutsch. Einmal setzt sich das Autorenteam um Rolf Böhm mit dem Kartennetzentwurf von Hajime Narukawa auseinander. Im anderen Kartenprojektionsbeitrag nähert sich Krisztián Kerkovits der Thematik mit mathematischer Exaktheit. Ferner können wir den Ausführungen von Gulay Pápay folgen, der sich mit dem Lebenswerk von Max Eckert beschäftigt hat.

Ich wünsche Ihnen viel Freude beim Lesen dieser Ausgabe!

Herzlichst,

$\mathrm{Ihr}$

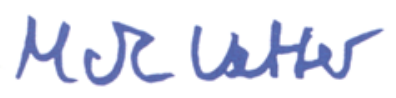

